\title{
A Note on Goldbach Partitions of Large Even Integers
}

\author{
Ljuben Mutafchiev \\ American University in Bulgaria \\ 2700 Blagoevgrad, Bulgaria \\ and \\ Institute of Mathematics and Informatics \\ Bulgarian Academy of Sciences, Bulgaria \\ ljuben@aubg.bg
}

Submitted: Jul 18, 2014; Accepted: Jan 30, 2015; Published: Feb 25, 2015

Mathematics Subject Classifications: 05A17, 11P32, 60C05, 60F05

\begin{abstract}
Let $\Sigma_{2 n}$ be the set of all partitions of the even integers from the interval $(4,2 n]$, $n>2$, into two odd prime parts. We show that $\left|\Sigma_{2 n}\right| \sim 2 n^{2} / \log ^{2} n$ as $n \rightarrow \infty$. We also assume that a partition is selected uniformly at random from the set $\Sigma_{2 n}$. Let $2 X_{n} \in(4,2 n]$ be the size of this partition. We prove a limit theorem which establishes that $X_{n} / n$ converges weakly to the maximum of two random variables which are independent copies of a uniformly distributed random variable in the interval $(0,1)$. Our method of proof is based on a classical Tauberian theorem due to Hardy, Littlewood and Karamata. We also show that the same asymptotic approach can be applied to partitions of integers into an arbitrary and fixed number of odd prime parts.
\end{abstract}

\section{Introduction and Statement of the Main Result}

For a given sequence of positive integers $\Lambda=\left\{\lambda_{1}, \lambda_{2}, \ldots\right\}$, by a $\Lambda$-partition of the positive integer $n$, we mean a way of writing it as a sum of positive integers from $\Lambda$ without regard to order; the summands are called parts. Let $\mathcal{P}=\left\{p_{1}, p_{2}, \ldots\right\}$ be the sequence of all odd primes arranged in increasing order. A prime partition is a $\Lambda$-partition with $\Lambda=\mathcal{P}$. Let $Q(n)$ be the number of prime partitions of $n$. Hardy and Ramanujan $[6,7]$ were apparently the first who studied the asymptotic behavior of the number of integer $(\Lambda=\{1,2, \ldots\})$ and prime partitions for large $n$. For prime partitions they proved the following asymptotic formula:

$$
\log Q(n) \sim 2 \pi \sqrt{\frac{n}{3 \log n}}, \quad n \rightarrow \infty .
$$


The study of the asymptotic behavior of $Q(n)$ itself is quite complicated. It turns out that the corresponding asymptotic formula contains transcendental sums over the primes which can be expressed in terms of zeros of the Riemann zeta function (for more details, see e.g. [9]; p. 240). Recently Vaughan [16] proposed and studied a modification of the problem, where $n$ is replaced by a continuous real variable. His asymptotic results avoid transcendental sums over primes.

Consider now the number $Q_{m}(n)$ of prime partitions of $n$ into $m$ parts $(1 \leqslant m \leqslant n)$. The bivariate generating function of the numbers $Q_{m}(n)$ is of Euler's type, namely,

$$
G(x, z)=1+\sum_{n=1}^{\infty} z^{n} \sum_{m=1}^{n} Q_{m}(n) x^{m}=\prod_{p_{k} \in \mathcal{P}}\left(1-x z^{p_{k}}\right)^{-1}
$$

(the proof may be found in [1]; Section 2.1). In this note we focus on the asymptotic behavior of the coefficients $Q_{2}(n)$ of $x^{2}$ and $z^{n}$ in the power series expansion of $G(x, z)$ in powers of $x$ and $z$. For $n>4, Q_{2}(n)$ counts the number of ways of representing $n$ as a sum of two odd primes. Obviously, $Q_{2}(n)=0$ if $n$ is odd. In 1742 Goldbach conjectured that $Q_{2}(n) \geqslant 1$ for every even integer $n>4$. This problem remains still unsolved (for more details, see e.g. [8]; Section 2.8 and p. 594). Another famous conjecture related to prime partitions was stated by Hardy and Littlewood [5], who predicted the asymptotic form of $Q_{2}(n)$ for large even $n$. They conjectured that

$$
\begin{aligned}
Q_{2}(n) & \sim 2 C_{2}\left(\prod_{p_{k} \in \mathcal{P}, p_{k} \mid n} \frac{p_{k}-1}{p_{k}-2}\right) \int_{2}^{n} \frac{d u}{\log ^{2} u} \\
& \sim 2 C_{2}\left(\prod_{p_{k} \in \mathcal{P}, p_{k} \mid n} \frac{p_{k}-1}{p_{k}-2}\right) \frac{n}{\log ^{2} n}, \quad n \rightarrow \infty,
\end{aligned}
$$

where $C_{2}$ is the twin prime constant

$$
C_{2}:=\prod_{p_{k} \in \mathcal{P}}\left(1-\frac{1}{\left(p_{k}-1\right)^{2}}\right)=0.6601618158 \ldots
$$

(for the role of $C_{2}$ in the distribution of the prime numbers, see again [8]; Section 22.20). This conjecture remains also still open.

In the present note we do not deal with the asymptotic equivalence (2) but consider the sum function

$$
S(2 n)=\sum_{2<k \leqslant n} Q_{2}(2 k), \quad n>2,
$$

counting all partitions of the even integers from the interval $(4,2 n]$ into two odd prime parts. Sometimes this kind of partitions are called Goldbach partitions. Let $\Sigma_{2 n}$ denote the set of these partitions. Our main result is the following asymptotic equivalence. 
Theorem 1. We have

$$
\left|\Sigma_{2 n}\right|=S(2 n) \sim \frac{2 n^{2}}{\log ^{2} n}, \quad n \rightarrow \infty .
$$

Consider now a random experiment. Suppose that we select a partition uniformly at random from the set $\Sigma_{2 n}$, i.e. we assign the probability $1 / S(2 n)$ to each Goldbach partition. We denote by $\mathbb{P}$ the uniform probability measure on $\Sigma_{2 n}$. Let $2 X_{n} \in(4,2 n]$ be the number that is partitioned by this random selection. $2 X_{n}$ is also called the size of this partition. Using Theorem 1, we determine the limiting distribution of the random variable $X_{n}$.

Theorem 2. If $0<u<1$, then

$$
\lim _{n \rightarrow \infty} \mathbb{P}\left(\frac{X_{n}}{n} \leqslant u\right)=u^{2}
$$

Remark 1. Using the Prime Number Theorem [8], Section 1.8, it is easy to show that the number of ordered pairs of primes not exceeding $2 n$ is also $\sim 2 n^{2} / \log ^{2} n$; cf. with the result of Theorem 1 . Hence, we conclude that almost all even integers that are $\leqslant 2 n$ have only one partition into two prime parts.

Remark 2. In probabilistic terms Theorem 2 shows that the typical size of a random Goldbach partition is a fraction of $2 n$. Moreover, Theorem 2 implies that $X_{n} / n$ converges weakly, as $n \rightarrow \infty$, to a random variable whose cumulative distribution function is

$$
F(u)=\left\{\begin{array}{lll}
0 & \text { if } & u \leqslant 0, \\
u^{2} & \text { if } & 0<u<1, \\
1 & \text { if } & u \geqslant 1
\end{array}\right.
$$

It can be easily seen that $F(u)$ is the distribution function of $\max \left\{U_{1}, U_{2}\right\}$, where $U_{1}$ and $U_{2}$ are two independent copies of a uniformly distributed random variable in the interval $(0,1)$.

Remark 3. One reason to study the sum function (3) is motivated by a result due to Brigham [2]. He has studied the asymptotic behavior of a similar sum function related to integer partitions weighted by the sequence of the von Mangoldt functions (the definition of a von Mangoldt function and its role in the proof of the Prime Number Theorem may be found in [8]; Section 17.7). The asymptotic behavior of a single term in Brigham's sum function was subsequently studied by Richmond [13] and Yang [17]. Their results are essentially based on Brigham's observations.

Remark 4. Another interesting problem on prime partitions is related to the asymptotic behavior of the coefficients $Q_{m}(n)$, the number of prime partitions of $n$ with $m$ parts (see (1)). Haselgrove and Temperley [9], p. 240, found an asymptotic form for $Q_{m}(n)$, 
whenever $m=m(n) \rightarrow \infty$ as $n \rightarrow \infty$ in a proper way. In probabilistic terms their result can be stated as follows. Consider a random variable, whose probability distribution function is defined by the ratio

$$
\frac{Q_{m}(n)}{Q(n)}, \quad m=1, \ldots, n
$$

Haselgrove and Temperley [9] showed that this random variable converges weakly to a nondegenerate random variable as $n \rightarrow \infty$. They also determined the moment generating function of this limiting variable. The asymptotic form of the mean and the variance of probability distribution (4) were found recently by Ralaivaosaona [12].

Our paper is organized as follows. Section 2 contains some preliminaries. The proofs of Theorems 1 and 2 are given in Section 3. Our method of proof is essentially based on a classical Tauberian theorem due to Hardy, Littlewood and Karamata (see [4]). Finally, in Section 4 we present an extension of our main result. In particular, we show that the same approach yields similar results for prime partitions of $n$ into $m>2$ parts whenever $m$ is fixed integer.

\section{Preliminary Results}

We start with a generating function identity for the sequence $\left\{Q_{2}(2 k)\right\}_{k>2}$ of the counts of Goldbach partitions.

Lemma 3. For any real variable $z$ with $|z|<1$, let

$$
f(z)=\sum_{p_{k} \in \mathcal{P}} z^{p_{k}}
$$

Then, we have

$$
2 \sum_{k>2} Q_{2}(2 k) z^{2 k}=f^{2}(z)+f\left(z^{2}\right) .
$$

Proof. Differentiating the left-hand side of (1) twice with respect to $x$ and setting then $x=0$ and $m=2$, we get

$$
\begin{aligned}
\left.\frac{\partial^{2} G(x, z)}{\partial x^{2}}\right|_{x=0, m=2} & =\left.\sum_{n=1}^{\infty} z^{n} \sum_{m=2}^{n} m(m-1) Q_{m}(n) x^{m-2}\right|_{x=0, m=2} \\
& =2 \sum_{n=1}^{\infty} Q_{2}(n) z^{n}=2 \sum_{k>2} Q_{2}(2 k) z^{2 k} .
\end{aligned}
$$

The last equality follows from the obvious identities $Q_{2}(1)=Q_{2}(2)=Q_{2}(4)=0$ and $Q_{2}(2 k+1)=0$ for $k=1,2, \ldots$. The right-hand side of (1) can be also written as 
$\exp \left(-\sum_{p_{k} \in \mathcal{P}} \log \left(1-x z^{p_{k}}\right)\right)$. Differentiating it twice, in the same way we find that

$$
\begin{aligned}
\left.\frac{\partial^{2} G(x, z)}{\partial x^{2}}\right|_{x=0}= & \left.\left(\exp \left(-\sum_{p_{k} \in \mathcal{P}} \log \left(1-x z^{p_{k}}\right)\right)\right)\left(\sum_{p_{k} \in \mathcal{P}} \frac{z^{p_{k}}}{1-x z^{p_{k}}}\right)^{2}\right|_{x=0} \\
& \quad+\left.\left(\exp \left(-\sum_{p_{k} \in \mathcal{P}} \log \left(1-x z^{p_{k}}\right)\right)\right)\left(\sum_{p_{k} \in \mathcal{P}} \frac{z^{2 p_{k}}}{\left(1-x z^{p_{k}}\right)^{2}}\right)\right|_{x=0} \\
= & f^{2}(z)+f\left(z^{2}\right),
\end{aligned}
$$

which completes the proof.

Further, we will use a Tauberian theorem by Hardy-Littlewood-Karamata whose proof may be found in [4]; Chapter 7. We use it in the form given by Odlyzko [11]; Section 8.2.

Hardy-Littlewood-Karamata Theorem. (See [11]; Theorem 8.7, p. 1225.) Suppose that $a_{k} \geqslant 0$ for all $k$, and that

$$
g(x)=\sum_{k=0}^{\infty} a_{k} x^{k}
$$

converges for $0 \leqslant x<r$. If there is $a \rho>0$ and a function $L(t)$ that varies slowly at infinity such that

$$
g(x) \sim(r-x)^{-\rho} L\left(\frac{1}{r-x}\right), \quad x \rightarrow r^{-},
$$

then

$$
\sum_{k=0}^{n} a_{k} r^{k} \sim\left(\frac{n}{r}\right)^{\rho} \frac{L(n)}{\Gamma(\rho+1)}, \quad n \rightarrow \infty .
$$

Remark. A function $L(t)$ varies slowly at infinity if, for every $u>0, L(u t) \sim L(t)$ as $t \rightarrow \infty$.

\section{Proof of the Main Result}

Proof of Theorem 1. We need to show that power series (6) satisfies the conditions of Hardy-Littlewood-Karamata theorem. The next lemma establishes an asymptotic equivalence of $f(z)$ as $z \rightarrow 1^{-}$.

Lemma 4. Let $f(z)$ be the power series defined by (5). Then, as $z \rightarrow 1^{-}$,

$$
f(z) \sim-\frac{1}{\left(\log \frac{1}{z}\right)\left(\log \log \frac{1}{z}\right)} .
$$


Proof. As usual, by $\pi(y)$ we denote the number of primes which do not exceed the positive real number $y$. In (5) we set $z=e^{-t}, t>0$, and apply an argument similar to that given by Stong [15] (see also [3]). We have

$$
f\left(e^{-t}\right)=\int_{0}^{\infty} e^{-y t} d \pi(y)=\int_{0}^{\infty} t e^{-y t} \pi(y) d y=\int_{0}^{\infty} \pi(s / t) e^{-s} d s=I_{1}(t)+I_{2}(t),
$$

where

$$
I_{1}(t)=\int_{0}^{t^{1 / 2}} \pi(s / t) e^{-s} d s, \quad I_{2}(t)=\int_{t^{1 / 2}}^{\infty} \pi(s / t) e^{-s} d s .
$$

For $I_{1}(t)$ we use the bound $\pi(s / t) \leqslant s / t$. Hence, for enough small $t>0$, we obtain

$$
\begin{aligned}
0 \leqslant I_{1}(t) \leqslant \frac{1}{t} \int_{0}^{t^{1 / 2}} s e^{-s} d s & =\frac{1}{t}\left(-\left.s e^{-s}\right|_{0} ^{t^{1 / 2}}+\int_{0}^{t^{1 / 2}} e^{-s} d s\right) \\
& =\frac{1}{t} O\left(t^{1 / 2}\right)=O\left(t^{-1 / 2}\right) .
\end{aligned}
$$

The estimate for $I_{2}(t)$ follows from the Prime Number Theorem with an error term given in a suitable form. So, it is known that, for $y>1$,

$$
\pi(y)=\frac{y}{\log y}+O\left(\frac{y}{\log ^{2} y}\right)
$$

(see e.g. [10]; Theorem 23, p. 65). Furthermore, for $s \geqslant t^{1 / 2}$, we have $\log s \geqslant-\frac{1}{2} \log \frac{1}{t}$. Hence, as in [15], we get

$$
\begin{aligned}
\pi(s / t) & =\frac{s}{t} \frac{1}{\log \frac{1}{t}+\log s}+O\left(\frac{s}{t\left(\log \frac{1}{t}+\log s\right)^{2}}\right) \\
& =\frac{s}{t \log \frac{1}{t}}\left(1+O\left(\frac{|\log s|}{\log \frac{1}{t}}\right)\right)+O\left(\frac{s}{t \log ^{2} \frac{1}{t}}\right) \\
& =\frac{s}{t \log \frac{1}{t}}+O\left(\frac{s(1+|\log s|)}{t \log ^{2} \frac{1}{t}}\right) .
\end{aligned}
$$

We also recall that in (10) we have used the obvious estimate

$$
\int_{0}^{t^{1 / 2}} s e^{-s} d s=O\left(t^{1 / 2}\right) .
$$


Combining (11) and (12), we obtain

$$
\begin{aligned}
I_{2}(t) & =\frac{1}{t \log \frac{1}{t}} \int_{t^{1 / 2}}^{\infty} s e^{-s} d s+O\left(\frac{1}{t \log ^{2} \frac{1}{t}} \int_{t^{1 / 2}}^{\infty} s(1+|\log s|) e^{-s} d s\right) \\
& =\frac{1}{t \log \frac{1}{t}}\left(\int_{0}^{\infty} s e^{-s} d s+O\left(t^{1 / 2}\right)\right)+O\left(\frac{1}{t \log ^{2} \frac{1}{t}}\right) \\
& =\frac{1}{t \log \frac{1}{t}}+O\left(\frac{1}{t^{1 / 2} \log \frac{1}{t}}\right)+O\left(\frac{1}{t \log ^{2} \frac{1}{t}}\right) \\
& \sim \frac{1}{t \log \frac{1}{t}}, \quad t \rightarrow 0^{+} .
\end{aligned}
$$

Hence, by (9), (10) and (13),

$$
f\left(e^{-t}\right) \sim \frac{1}{t \log \frac{1}{t}}, \quad t \rightarrow 0^{+}
$$

The proof is now completed after the substitution $t=\log \frac{1}{z}$.

Since

$$
\log \frac{1}{z}=-\log z=-\log (1-(1-z)) \sim 1-z, \quad z \rightarrow 1^{-},
$$

the asymptotic equivalence in Lemma 4 becomes

$$
f(z) \sim \frac{1}{(1-z) \log \frac{1}{1-z}}, \quad z \rightarrow 1^{-} .
$$

Therefore,

$$
f^{2}(z)+f\left(z^{2}\right) \sim \frac{1}{(1-z)^{2} \log ^{2} \frac{1}{1-z}}, \quad z \rightarrow 1^{-},
$$

which implies that the series $\sum_{k>2} Q(2 k) z^{2 k}$ satisfies condition (7) of Hardy-LittlewoodKaramata Tauberian theorem with $r=1, \rho=2$ and $L(t)=\frac{1}{\log ^{2} t}$ (see also (6)). The asymptotic equivalence of Theorem 1 follows immediately from (8).

Proof of Theorem 2. Recall that $2 X_{n} \in(4,2 n]$ equals the size of a Goldbach partition that is chosen uniformly at random from the set $\Sigma_{2 n}$ of all such partitions. Since $S(2 n)=\left|\Sigma_{2 n}\right|$ and, for any $N \in(2, n], S(2 N)=\left|\Sigma_{[2 N]}\right|$ (where $[a]$ denotes the integer part of the real number $a$ ), from (3) it follows that

$$
\mathbb{P}\left(2 X_{n} \leqslant 2 N\right)=\frac{S(2 N)}{S(2 n)} .
$$

Setting $N \sim u n, 0<u<1$, and applying Theorem 1 twice - to the numerator and the denominator of (14), we see that the limit of (14), as $n \rightarrow \infty$, is $u^{2}$. This completes the proof. 


\section{Prime Partitions with More Than Two Parts}

Let $m>2$ be an integer and let $\Sigma_{m, n}$ denote the set of prime partitions of the integers from the interval $(4, n]$ into $m$ parts. The goal of this section is to extend the results of Theorems 1 and 2 to prime partitions from the class $\Sigma_{m, n}$. We state them below as two separate theorems.

Theorem 5. For any fixed integer $m>2$, we have

$$
\left|\Sigma_{m, n}\right| \sim \frac{1}{m !}\left(\frac{n}{\log n}\right)^{m}, \quad n \rightarrow \infty
$$

Furthermore, let $X_{m, n}$ denote the size of a prime partition selected uniformly at random from the class $\Sigma_{m, n}$. (The uniform probability measure on $\Sigma_{m, n}$ is again denoted by $\mathbb{P}$.)

Theorem 6. If $0<u<1$ and $m$ is as in Theorem 5, then

$$
\lim _{n \rightarrow \infty} \mathbb{P}\left(\frac{X_{m, n}}{n} \leqslant u\right)=u^{m}
$$

Theorem 6 shows a weak convergence similar to that established in Theorem 2 . Namely, for any fixed integer $m, X_{m, n} / n$ converges, as $n \rightarrow \infty$, to $\max \left\{U_{1}, \ldots, U_{m}\right\}$, where $U_{1}, \ldots, U_{m}$ are independent copies of a random variable that is uniformly distributed in the interval $(0,1)$.

Below we only sketch the proof of Theorem 5. The proof of Theorem 6 is almost identical to that of Theorem 2.

Proof of Theorem 5. (Sketch.) Our main tool is again the generating function identity (1). We notice first that the coefficients $Q_{m}(n)$ are $=0$ if either $m$ is odd and $n$ is even or $m$ is even and $n$ is odd. By the definition of $Q_{m}(n)$, we also have

$$
\left|\Sigma_{m, n}\right|=\sum_{k \leqslant n} Q_{m}(k)
$$

We compute the $m$ th derivative of the infinite product in (1) using Faa di Bruno formula for derivatives of compound functions (see e.g. [14]; Section 2.8). We introduce the following auxiliary notations:

$$
\begin{aligned}
& b(x)=b(x, z):=-\sum_{p_{k} \in \mathcal{P}} \log \left(1-x z^{p_{k}}\right), \\
& b_{j}=b_{j}(x, z):=\frac{\partial^{j} b(x, z)}{\partial x^{j}}, j=1, \ldots, m .
\end{aligned}
$$

Using formulae (43) and (46) of [14]; Section 2.8, we obtain

$$
\frac{d^{m}}{d x^{m}} e^{b(x)}=e^{b(x)} b_{1}^{m}+R_{m}
$$


where

$$
R_{m}=R_{m}(x, z)=e^{b(x, z)} \widetilde{\sum} \frac{m !}{k_{1} ! \cdots k_{m} !}\left(\frac{b_{1}}{1 !}\right)^{k_{1}} \cdots\left(\frac{b_{m}}{m !}\right)^{k_{m}}
$$

and $\widetilde{\sum}$ denotes the sum over all integers $k_{j} \geqslant 0, j=1, \ldots, m$, such that $\sum_{j=1}^{m} j k_{j}=m$ and $k_{1}<m$. Setting $x=0$ in (16), we find that $b(0, z)=0$ and $b_{j}(0, z)=f\left(z^{j}\right), j=1, \ldots, m$, where the function $f(z)$ is defined (5). Moreover, in the right-hand side of (18) we have $k_{1}+\cdots+k_{m} \leqslant m-1$. In fact, since $k_{1}<m$ by the definition of $\widetilde{\sum}$ at least one $k_{j}$ is $>0$ for $j \geqslant 2$. Hence if $m=k_{1}+k_{2}+\cdots+k_{m}$, then $m<k_{1}+\sum_{j=2}^{m} j k_{j}=m$. Since $f\left(z^{j}\right)=O(f(z))$ as $z \rightarrow 1^{-}$and since $k_{1}+\cdots+k_{m} \leqslant m-1$, we conclude that $R_{m}(0, z)=O\left(f^{m-1}(z)\right)$. Therefore (17) becomes

$$
\frac{d^{m}}{d x^{m}} e^{b(x)}=f^{m}(z)+O\left(f^{m-1}(z)\right)
$$

or, equivalently,

$$
\left.\frac{\partial^{m} G(x, z)}{\partial x^{m}}\right|_{x=0}=f^{m}(z)+O\left(f^{m-1}(z)\right)
$$

as $z \rightarrow 1^{-}$. On the other hand,

$$
\left.\frac{\partial^{m} G(x, z)}{\partial x^{m}}\right|_{x=0}=m ! \sum_{k \geqslant m} Q_{m}(k) z^{k}
$$

Applying Lemma 4, as in the proof of Theorem 1, we obtain the asymptotic equivalence

$$
f^{m}(z) \sim \frac{1}{(1-z)^{m} \log ^{m} \frac{1}{1-z}}, \quad z \rightarrow 1^{-} .
$$

The observations in (19)-(21) imply that

$$
\left.\frac{\partial^{m} G(x, z)}{\partial x^{m}}\right|_{x=0} \sim \frac{1}{(1-z)^{m} \log ^{m} \frac{1}{1-z}}, \quad z \rightarrow 1^{-} .
$$

So, condition (7) of Hardy-Littlewood-Karamata theorem is satisfied with $r=1, \rho=m$ and $L(t)=\frac{1}{\log ^{m} t}$. The required result follows at once from (15) and (8).

\section{Acknowledgements}

I am grateful to the referee for carefully reading the paper and for their helpful comments and suggestions.

\section{References}

[1] G.E. Andrews. The Theory of Partitions. Encyclopedia Math. Appl., no. 2. AddisonWesley, Reading, MA, 1976. 
[2] N.A. Brigham. On a certain weighted partition function. Proc. Amer. Math. Soc., 1:192-204, 1950.

[3] P. Erdős and P. Turán. On some problems of a statistical group theory, IV. Acta Math. Acad. Sci. Hungar., 19:413-435, 1968.

[4] G.H. Hardy. Divergent Series. Oxford Univ. Press, Oxford, 1949.

[5] G.H. Hardy and J.E. Littlewood. Some problems of "Partitio Numerorum", III: On the expression of a number as a sum of primes. Acta Math., 44:1-70, 1922.

[6] G.H. Hardy and S. Ramanujan. Asymptotic formulae for the distribution of integers of various types. Proc. London Math. Soc., 16:112-132, 1917.

[7] G.H. Hardy and S. Ramanujan. Asymptotic formulae in combinatory analysis. Proc. London Math. Soc., 17:75-115, 1918.

[8] G.H. Hardy and E.M. Wright. An Introduction to the Theory of Numbers. Oxford Univ. Press, Oxford, 2008.

[9] C.B. Haselgrove and H.N.V. Temperley. Asymptotic formulae in the theory of partitions. Proc. Cambr. Phil. Soc., 50:225-241, 1954.

[10] A.E. Ingham. The Distribution of the Prime Numbers. Cambridge Univ. Press, Cambridge, 1990.

[11] A.M. Odlyzko. Asymptotic enumeration methods. In Handbook of Combinatorics (R. Graham, M. Grötschel and L. Lovász, Eds.), volume II, pages 1063-1229, Elsevier, 1995.

[12] D. Ralaivaosaona. On the number of summands in a random prime partition, Monatsh. Math., 166:505-524, 2012.

[13] L.B. Richmond. A general asymptotic result for partitions. Canad. J. Math., 27:10831091, 1975.

[14] J. Riordan. An Introduction to Combinatorial Analysis. Wiley, New York, 1958.

[15] R. Stong. The average order of a permutation. Electron. J. Combin., 5:\#R41, 1998.

[16] R.C. Vaughan. On the number of partitions into primes. Ramanujan J., 15:109-121, 2008.

[17] Y. Yang. Partitions into primes. Trans. Amer. Math. Soc., 352:2581-2600, 2000. 